\title{
Budget impact of sequential treatment with first-line afatinib versus first-line osimertinib in non-small-cell lung cancer patients with common EGFR mutations
}

\author{
Lotte Westerink $k^{1,2,10} \mathbb{0} \cdot$ Jelmer L. J. Nicolai ${ }^{3} \cdot$ Carl Samuelsen $^{4} \cdot$ Hans J. M. Smit ${ }^{5} \cdot$ Pieter E. Postmus ${ }^{6}$. \\ Ingolf Griebsch ${ }^{7}$ Maarten J. Postma ${ }^{1,8,9}$
}

Received: 6 September 2019 / Accepted: 3 April 2020 / Published online: 23 April 2020

(c) The Author(s) 2020

\begin{abstract}
Background The therapeutic landscape for non-small-cell lung cancer (NSCLC) patients that have common epidermal growth factor receptor (EGFR) mutations has changed radically in the last decade. The availability of these treatment options has an economic impact, therefore a budget impact analysis was performed.

Methods A budget impact analysis was conducted from a Dutch healthcare perspective over a 5-year time horizon in EGFR-mutant NSCLC patients receiving first-line afatinib $\left(\right.$ Gilotrif $^{\circledR}$ ) versus first-line osimertinib $\left(\right.$ Tagrisso ${ }^{\circledR}$ ), followed by subsequent treatments. A decision analysis model was constructed in Excel. Scenario analyses and one-way sensitivity analysis were used to test the models' robustness.

Results Sequential treatment with afatinib versus first-line treatment with osimertinib showed mean total time on treatment (ToT) of 29.1 months versus 24.7 months, quality-adjusted life months (QALMs) of 20.2 versus 17.4 with mean cost of $€ 108,166$ per patient versus $€ 143,251$ per patient, respectively. The 5-year total budget impact was $€ 110.4$ million for the afatinib sequence versus $€ 158.6$ million for the osimertinib sequence, leading to total incremental cost savings of $€ 48.15$ million.

Conclusions First-line afatinib treatment in patients with EGFR-mutant NSCLC had a lower financial impact on the Dutch healthcare budget with a higher mean ToT and QALM compared to osimertinib sequential treatment.
\end{abstract}

Keywords Budget impact · Afatinib · Osimertinib · Treatment sequencing $\cdot$ I15 $\cdot$ I18

JEL Classification I15 $\cdot$ I18

Electronic supplementary material The online version of this article (https://doi.org/10.1007/s10198-020-01186-9) contains supplementary material, which is available to authorized users.

Lotte Westerink

lotte.westerink@ascacademics.com

1 Department of Health Sciences, University Medical Center Groningen, University of Groningen, Groningen, The Netherlands

2 Asc Academics Inc., New York, USA

3 Boehringer Ingelheim BV, Alkmaar, The Netherlands

4 Outcomes Analytica AS, Oslo, Norway

5 Rijnstate Hospital Arnhem, Arnhem, The Netherlands

6 Department of Pulmonary Diseases, University Medical Centre, Leiden, The Netherlands
7 Boehringer Ingelheim International $\mathrm{GmbH}$, Ingelheim, Germany

8 Unit of Pharmacotherapy, -Epidemiology and -Economics, Department of Pharmacy, University of Groningen, Groningen, The Netherlands

9 Department of Economics, Econometrics and Finance, Faculty of Economics and Business, University of Groningen, Groningen, The Netherlands

1012 East 49th Street, New York, NY 10017, USA 


\section{Background}

Lung cancer is the worldwide leading cause of cancer mortality [1]. Non-small-cell lung cancer (NSCLC) is the most common type with $40 \%$ of the patients presenting with adenocarcinoma [2] Approximately 10-20\% of Caucasian patients with non-resectable lung adenocarcinoma have somatic mutations of the epidermal growth factor receptor (EGFR) gene. The therapeutic landscape for patients with advanced EGFR mutation-positive NSCLC (exon 19 deletion and L858R) has changed radically with EGFR tyrosine kinase inhibitors (TKIs) becoming the new first-line standard treatment instead of chemotherapy. Data from multiple phase III randomized clinical trials (RCTs) have shown that the first-generation EGFR TKIs, erlotinib or gefitinib, and the second-generation EGFR TKI afatinib improved progression-free survival (PFS) and quality of life (QoL) compared to standard platinum-based chemotherapy [3-9]. More recent trials suggested that secondgeneration EGFR TKIs (afatinib and dacomitinib) may be more effective than first-generation agents $[10,11]$. Recently, a third-generation TKI, osimertinib, has shown improved PFS compared to first-generation TKIs [12].

Eventually, patients treated with first-line EGFR TKIs develop resistance to treatment. The majority of patients (50-70\%) progressing on first- and second- generation TKIs, develop secondary mutations such as the T790M point mutation. For patients with T790M-positive tumors, osimertinib is an approved second-line treatment based on improved PFS compared to standard of care observed in the AURA3 study [13]. ESMO guidelines recommended T790M mutation testing in all patients progressing on first-line TKI treatment [2]. For patients progressing on first-line therapy without T790M mutation, guidelines described complex and heterogeneous molecular mechanisms of resistance, for example, MET or HER2 amplification, BRAF or KRAS mutations, and small cell transformation. For these patients, the current standard treatment is platinum-based doublet chemotherapy (PDC). When patients progress on first-line osimertinib, the most frequent resistance mechanisms are MET amplification (15\%) and EGFR C797S mutation (7\%) [14]. There are currently no targeted treatment options available after osimertinib and patients failing first-line osimertinib treatment will receive chemotherapy or best supportive care $[15,16]$.

There is increasing evidence suggesting that patients treated with first-line afatinib are well suited for subsequent treatment with osimertinib. In a retrospective pooled analysis of data from 34 patients with common sensitizing EGFR mutations treated in the LUX-Lung 3, 6 and 7 studies, the median time on sequential treatment with osimertinib after first-line afatinib was 31.5 months [17]; the majority of patients were treated with osimertinib in the third, or later, line setting. Furthermore, a global observational real-world study showed that the median time on treatment for sequential first-line afatinib followed by second-line osimertinib was 27.6 months [18].

Due to the high number of life years lost by early death, lung cancer has one of the highest burdens of disease in the Netherlands $[19,20]$. The economic impact of a first-line treatment with afatinib followed by re-biopsy guided osimertinib therapy versus treatment of first-line osimertinib followed by subsequent chemotherapy has not been assessed in the Netherlands. In this study, the medical cost of treatment and the budget impact of these different treatment options for patients with common EGFR mutations were evaluated.

\section{Materials and methods}

\section{Model design}

A decision-analytic model was constructed in Microsoft Excel to assess the budget impact of different first-line treatment approaches in advanced or metastatic NSCLC patients with common EGFR mutations. The model compared two treatment strategies (Fig. 1): first-line afatinib (Gilotrif ${ }^{\circledR}$ ) followed by second-line osimertinib (Tagrisso $\left.{ }^{\circledR}\right)$ (T790M positive on progression) or chemotherapy (T790M negative on progression) followed by third-line chemotherapy versus first-line treatment osimertinib followed by second-line chemotherapy. Patients are assumed to go into the health states best supportive care (BSC) or death after failing chemotherapy. The analysis was performed from the Dutch healthcare perspective. The model estimated outcomes and costs for each treatment approach over a 5-year period. This analysis was developed in accordance with the International Society for Pharmacoeconomics and Outcomes Research's good practices for budget impact analysis [21].

This budget impact analysis represents a base case. Oneway deterministic sensitivity analysis and scenario analysis are performed to determine the influence of uncertainty around model inputs.

\section{Model inputs}

\section{Study population}

The eligible population included individuals with metastatic NSCLC whose tumors have EGFR deletion 19 or L858R mutations initiating first-line treatment. According to the Dutch Cancer Registration, there were 9175 patients with NSCLC in 2017 [20]. Approximately 10-20\% of Caucasian patients with non-resectable lung adenocarcinoma have somatic mutations of the EGFR gene [2], leading to 


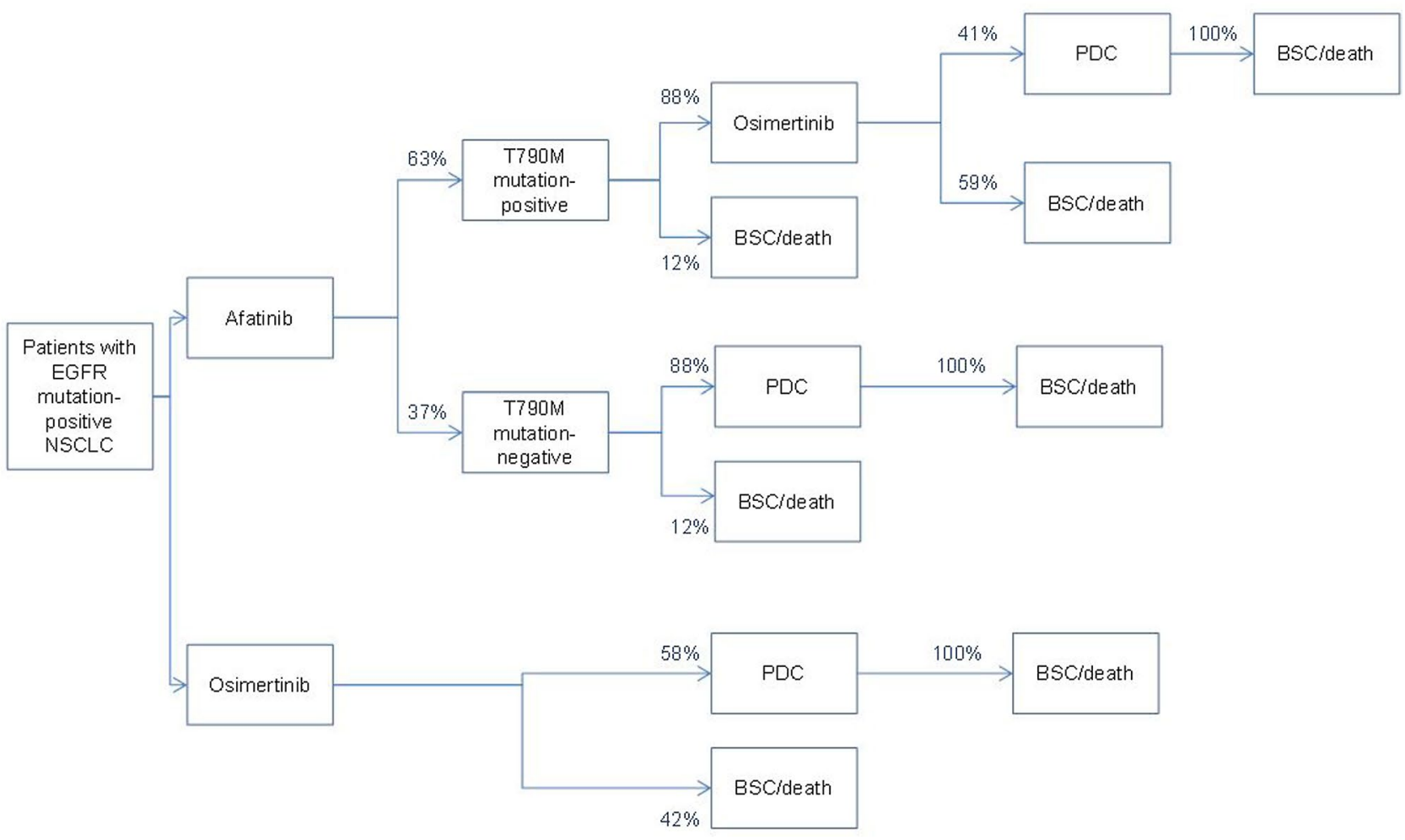

Fig. 1 Decision-analytic model to assess the budget impact of firstline treatment with afatinib versus osimertinib in EGFR mutationpositive NSCLC patients. BSC best supportive care, EGFR epidermal growth factor receptor, $N S C L C$ non-small-cell lung cancer, $P D C$ platinum doublet chemotherapy

trial demonstrated a significantly longer median PFS of 18.9 months with osimertinib versus standard EGFR TKIs gefitinib and erlotinib with a median PFS of 10.2 months [12].

Eventually the majority of patients will develop resistance to first-line treatment, mostly by developing secondary mutations such as the T790M point mutation. Data from the AURA3 [13] and IMPRESS [22] studies was used for second-line osimertinib and chemotherapy, respectively. In the AURA3 trial, osimertinib showed a significant improvement in median PFS at 10.1 months versus 4.4 months for chemotherapy (pemetrexed plus either carboplatin or cisplatin) in T790 mutation-positive advanced NSCLC patients. Furthermore in second-line treatment with chemotherapy, the IMPRESS trial showed that continuation of a standard EGFR TKI did not prolong PFS in patients who received PDC as a subsequent line of treatment [22]. Both treatment arms showed a median PFS of 5.4 months. For this reason, there will be no continuation of EGFR TKI treatment when chemotherapy is the second-line treatment. Because there is limited data on the efficacy of third-line chemotherapy, we assumed similar efficacy to second-line chemotherapy. advanced NSCLC patients were applied. The FLAURA 
The demographic characteristics of patients included in the RCTs of LUX-Lung 3, FLAURA, AURA3 and IMPRESS (published between 2015 and 2018) were well balanced [8, 12, 13, 22]. All patients included concerned adult patients with histological confirmed lung adenocarcinoma, advanced or metastatic stage IIIB and IV with mainly the following common EGFR mutations: exon 19 deletion and exon $21 \mathrm{~L} 858 \mathrm{R}$ point mutation (further mutation details are provided in Tables 4-7 in the Supplementary Data). The median age in the RCTs ranged from 60 to 64 years old and the distribution of female ranged $54-65 \%$. The distribution of race ranged $32-36 \%$ for white, $62-79 \%$ for Asian and $1-3 \%$ other. The studies LUX-Lung 3 and IMPRESS did not elaborate on the distribution between white and other, therefore a range of $21-28 \%$ concerns a combination of both for these studies. The WHO performance status 0 ranged from 40 to $41 \%$ and performance status 1 from 59 to $60 \%$. A WHO performance status of 0 indicates that the patient is fully active and able to carry out all predisease activities without restrictions and a WHO performance status of 1 indicates that the patient is restricted in physically strenuous activity but is ambulatory and able to carry out work of a light or sedentary nature, such as light office work [12]. Only the WHO performance status was not taken into account in the AURA3 study. The distribution of patient's history of smoking was $65-67 \%$ for never, $30-32 \%$ for former and $2-8 \%$ for current. In both the AURA3 and IMPRESS studies, it was unknown what the percentage of former and current smokers was.

To ensure that survival data for patients with similar characteristics were used in the model, the model and analyses were limited to patients with common mutations. A network meta-analysis (NMA) was performed, following a Bayesian framework with two designed and estimated networks. The description of the performed NMA is included in the Supplementary Data. WebPlotDigitizer was used to extract the Kaplan-Meier data from the publications, $\mathrm{R}$ version 3.5.1 and the SurvHE package was used to generate pseudoindividual patient-level data [23]. The partitioned survival model with a Weibull distribution for the common mutations estimated the proportion of a cohort in each state. The modeled PFS values were then used as proxy to estimate time on treatment for each intervention (Table 1).

\section{Transition probabilities}

Total time in progression-free disease and post-progression treatment options per treatment plan were calculated using a decision tree combining survival data and prescription data (Fig. 1). Mean survival time from survival modeling was used to calculate the expected time on treatment for the different interventions. In addition, data from realworld studies were used in the model [24, 25]. Prevalence of detectable T790M rates depend on available diagnostic testing technology and have been reported to range from 50 to $70 \%[17,18,26]$. For patients treated with afatinib, a $63 \%$ probability of a T790M mutation was used in the base case [25]. Estimates of the proportions of patients receiving subsequent therapy were taken from the literature. In the LUX-Lung trials, $71 \%$ of the intention-to-treat population and $88 \%$ of patients from countries with universal reimbursement received subsequent treatment (Table 1) [17]. In FLAURA, 58\% of patients progressing on first-line osimertinib received subsequent therapy and in AURA III $41 \%$ of patients with T790M mutation-positive disease who progressed on second-line osimertinib received subsequent treatment $[12,13]$.

Due to limited availability of overall survival data for the modeled interventions, we have assumed that patients not receiving subsequent therapy following progression will continue to progress and receive best supportive care and/ or die.

\section{Utilities}

To be able to include adverse events into the budget impact model, disutilities related to specific adverse events in patients with NSCLC [27] were combined with data from health-related quality of life (HRQoL) in patients with NSCLC in different health states [28] (Table 1). The model considered ten grade $\geq 3$ adverse events associated with treatment. Assumptions were made for dyspnea, leukopenia, paronychia, stomatitis based on available data [27]. For example, the utility of leukopenia was assumed to be the same as the utility of neutropenia. Table 1 gives an overview of all (dis)utilities, sources and assumptions.

Comparing the utility in progression-free $(\mathrm{PF})$ patients on first-line treatment with PF patients on second-line treatment resulted in a slightly higher but not clinically relevant mean utility in patients on second-line treatment [28]. One potential explanation for this could be that patients who move on to a higher line of treatment comprise a subset that is relatively fit [28].

\section{Costs}

An overview of all healthcare costs used in the model is shown in Table 1. The base-case analysis was the Dutch healthcare perspective; only direct medical costs were considered. The costs of both afatinib and osimertinib are based on the price for a monthly prescription. The mean cost for having grade 3 adverse events was calculated per patient for every specific treatment step, irrespective of time on treatment. In the model, every patient starting a specific treatment step will have a mean cost for grade 3 adverse events irrespective of time on treatment. The cost of a T790M 
Table 1 Overview of model parameters included in the budget impact model for sequential treatment with afatinib versus first-line osimertinib in NSCLC patients with common EGFR mutations

\begin{tabular}{lllll} 
Mean Afatinib & Osimertinib & PDC & BSC/death & Source and assumptions \\
\cline { 2 - 3 } & First-line Second-line & &
\end{tabular}

\section{Transition probabilities—afatinib treatment arm}

T790M mutation-positive

First-line
Second-line
Third-line
Fourth-line
T790M mutation-negative
First-line

$\begin{array}{lll}- & 63 \% & - \\ - & - & 88 \% \\ - & - & - \\ - & - & -\end{array}$

First-line

Second-line

Third-line

Transition probabilities—osimertinib treatment arm

First-line

Second-line

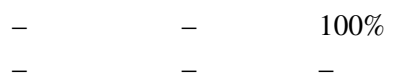

Third-line

Disutilities and incidences of grade $\geq \mathbf{3}$ adverse events

Anaemia
Diarrhoea
Fatigue

Neutropenia

Rash

Dyspnea

Leukopenia

Paronychia

Stomatitis

\section{Health states}

\section{First-line}

Second-line

Third-line

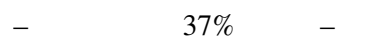

- $\quad-\quad \quad-$

$-$

$100 \%$

$-$

$-$

$\begin{array}{lll}-0.07346 & 0.44 \% & 1.08 \% \\ -0.0468 & 14.41 \% & 2.15 \% \\ -0.07346 & 1.31 \% & 0.72 \% \\ -0.0897 & 0.44 \% & - \\ -0.03248 & 16.16 \% & 1.08 \% \\ -0.07346 & - & 0.36 \% \\ -0.0897 & 0.44 \% & - \\ -0.03248 & 11.35 \% & 0.36 \% \\ -0.0468 & 8.73 \% & 0.36 \%\end{array}$

$\begin{array}{lll}0.710 & 0.688 & 0.707 \\ 0.730 & 0.708 & 0.727 \\ 0.620 & 0.598 & 0.617\end{array}$

Simulated mean PFS from survival model

First-line
Second-line
Third-line

-line

\section{Costs}

Treatment costs/month

T790M mutation testing

\section{Adverse events costs}

Anemia

Leukopenia

Neutropenia

Diarrhea

Rash

Dyspnea

Stomatitis

Fatigue

Paronychia

Total cost per treatment for adverse events

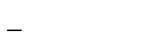

$-$

$-$

$-$

$€ 157.02$

$€ 1945.59$
$€ 1935.48$
$€ 1399.53$
$€ 1500.97$
$€ 87.99$
$€ 827.16$
$€ 1330.85$
$€ 83.63$
$€ 2.21$

$€ 2.21$

$\begin{array}{ll}16.99 & 20.80 \\ - & 15.23\end{array}$

$€ 2440.1$

(1)

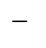

$$
€ 618
$$

$€ 8.50$

$€ 20.92$

$€ 8.45$

$€ 6.11$

$€ 216.30$

$€ 14.22$

$-$

$€ 116.23$

$€ 1.05$

$€ 0.25$

$€ 371.11$
- $\quad-$

- $\quad-\quad 12 \%$

\begin{tabular}{l}
$-\quad 41 \% \quad 59 \%$ \\
\hline
\end{tabular}

- $\quad-\quad 100 \%$

- $\quad-$

- $\quad 88 \% \quad 12 \%$

$\begin{array}{lll}- & - & 100 \%\end{array}$

[25]

Assumption

[13]

Assumption

[25]

[10, 13]

Assumption$$
\text { [12] }
$$

Assumption

$\begin{array}{lll}- & - & 100 \%\end{array}$

$\begin{array}{lll}0.72 \% & 6.31 \% & - \\ 1.08 \% & - & - \\ 1.08 \% & 12.61 \% & - \\ 1.43 \% & 18.02 \% & - \\ 0.72 \% & - & - \\ 1.08 \% & - & - \\ - & 8.11 \% & - \\ - & - & - \\ - & 0.90 \% & -\end{array}$

Assumption based on disutilities from Nafees et al. [27] $\begin{array}{llll}0.672 & - & {[8,12,13,21,26,27]} & - \\ 0.692 & - & - & - \\ 0.582 & - & - & -\end{array}$

$[8,12]$

$6.71-$ [13]

$6.71-[22]$

[31, 35, 36]

[37]

[38]

$-$

[30]

$-$

[40]

[41]

[36]

adverse 
Table 1 (continued)

Base case (range of sensitivity analysis)

Input data for one-way sensitivity analysis

Prevalence T790M (\%)

$63(52.5-73.0)$

Subsequent treatment T790M mutation positive (\% patients)

$88.0(70.4-100.0)$

Subsequent treatment after first-line osimertinib (\% patients)

$58.0(46.4-80.0)$

Afatinib cost $(€)$

$2440.15(1952.12-2928.18)$

Osimertinib cost $(€)$

6181.33 (4945.07-7417.6)

PDC cost $(€)$

$3679.04(2943.23-4414.85)$

T790M test cost $(€)$

157.02 (125.61-188.42)

Afatinib AE cost (€)

371.15 (296.89-445.33)

Osimertinib first-line AE cost $(€)$

$62.46(49.97-74.96)$

Osimertinib second-line AE cost $(€)$

$60.58(48.46-72.65)$

PDC AE cost $(€)$

$554.33(443.46-665.2)$

$A E$ adverse event, $B S C$ best supportive care, $P D C$ platinum doublet chemotherapy, $P F S$ progression-free survival

mutation test was taken into account for all patients progressing on first-line afatinib, as this is necessary to be eligible for sequential treatment with osimertinib.

\section{Model outputs}

The budget impact analysis estimated the costs associated with each treatment approach. Costs were reported as annual total costs for the population and as quality-adjusted life months (QALMs).

In accordance with the ISPOR guidelines for budget impact analysis [21], the robustness of model assumptions and uncertainty around specific parameters were tested in one-way sensitivity analysis. These variables included the prevalence of the T790M mutation, subsequent treatment ratio's after positive T790M mutation test, treatment cost for afatinib, osimertinib or PDC, respectively, and adverse event costs for different treatments. Model parameters mostly varied by $20 \%$ with a maximum of $100 \%$ in the base-case value. Due to the high reported variance in detected T790M mutations and recent data pointing towards heterogeneity between the first- and second-generation TKIs in tumor clonality and development of resistance mechanisms, sensitivity analyses were also performed to investigate how variance in detected T790M rates affected the model outcomes [29]. Results were displayed on tornado diagrams, ranked from the most sensitive to least sensitive parameters.

Scenario analyses were performed to explore alternative assumptions in line with the suggestion of the ISPOR guidelines for budget impact analysis [21]. A scenario analysis was performed using the log-normal distribution for the fitted patient survival data (compared to the Weibull distribution used in the base case) (Scenario A). A second scenario analysis was performed using a higher, real-world estimate (73.1\%) of the proportion of patients treated with afatinib that developed a T790M mutation at progression on first-line treatment (Scenario B) [24]. Also, in line with the ISPOR guidelines for budget impact analysis, we did not elaborate confidence intervals for the scenarios in a formal statistical manner. Notably, scenarios are designed with a specific purpose to inform the decision maker without the idea to potentially compare them statistically. The rules of inference have limited relevance to the decisions which budget impact models seek to inform and decisions in this context are based on the mean net benefits in the scenarios [30].

\section{Results}

Outcomes survival analysis from the Weibull distribution in decision-analytic model for the various treatment paradigms is shown in Fig. 2. An overview of the resulting mean time on treatment from each treatment strategy, percentage of patients receiving subsequent therapies, as well as estimated QALM and total average costs per patient is shown in Table 1 in the Supplementary data. Overall, sequential treatment with first-line afatinib had a mean total time on treatment of 29.1 months, QALM of 20.2 with a mean cost of $€ 108,166$ per patient, in contrast to the treatment group with first-line osimertinib treatment that had a mean total time on treatment of 24.7 months, QALM of 17.4 for a mean cost per patient of $€ 143,251$. Treating with afatinib as first-line treatment would lead to an incremental time on treatment of 4.5 months and incremental QALM of 2.8, together with a lower cost of $€ 35,085$ for total mean cost per patient versus first-line treatment with osimertinib. 


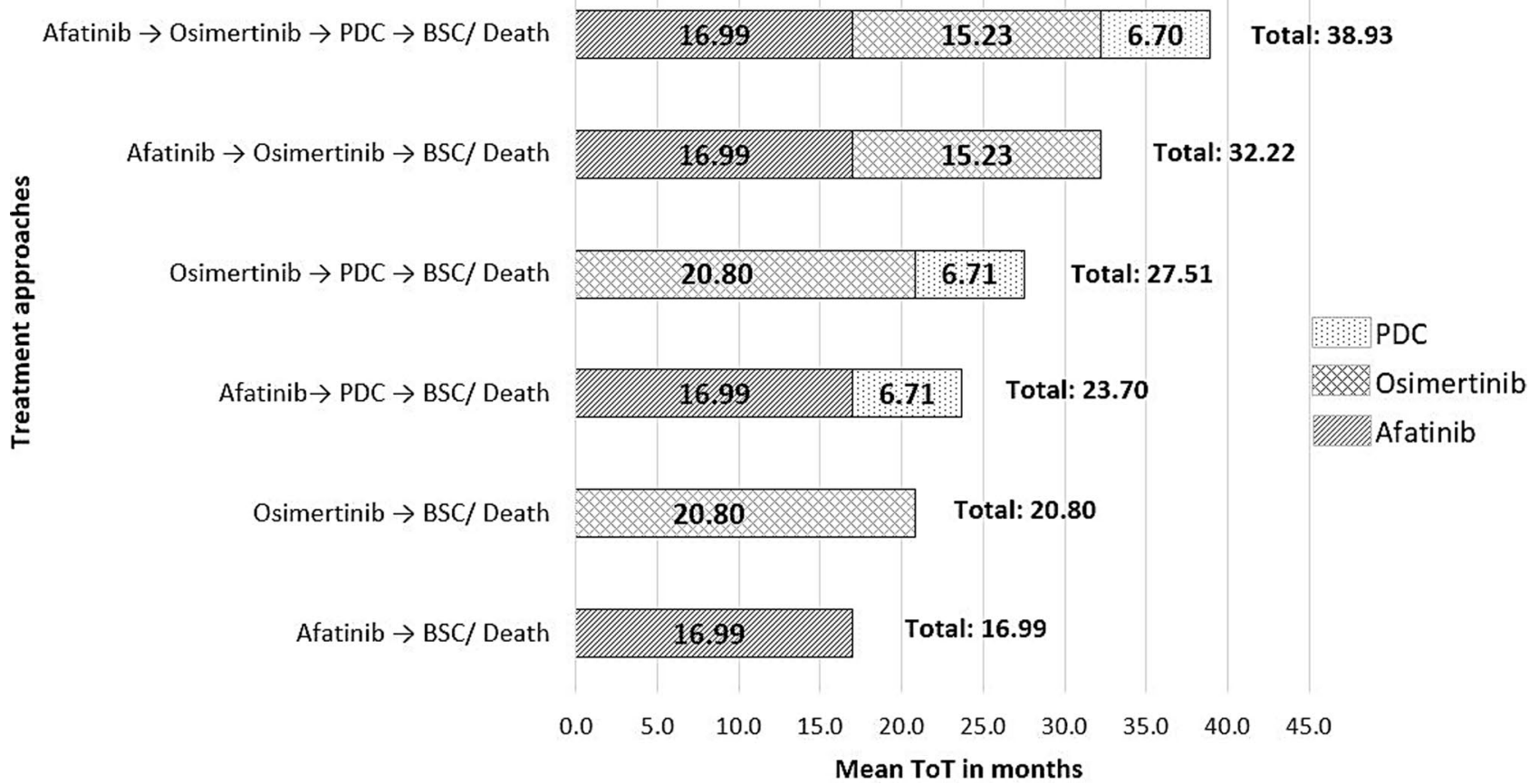

Fig. 2 Outcomes survival analysis with Weibull distribution in decision-analytic model: mean total time on treatment for patients by treatment paradigms. $B S C$ best supportive care, $P D C$ platinum doublet chemotherapy

\section{Budget impact analysis}

Costs of each first-line treatment strategy were based on number of packages per year (a package is referred as one treatment cycle per patient; see appendix). The total budget impact of each treatment sequence is shown in Fig. 3. For the treatment sequence starting with afatinib, the total budget impact over 5 years was $€ 110.4$ million, with total costs per year ranging from $€ 5.3$ million in year 1 to $€ 34.0$ million for year 5. Introduction of osimertinib as second-line therapy in this strategy was responsible for $46 \%$ of the total cost in year 3 and increased proportionally over time.

For the treatment sequence starting with osimertinib, the total budget impact over 5 years was $€ 158.6$ million, with total costs per year ranging from $€ 11.34$ million in year 1 , increasing to $€ 36.98$ million for the 3rd year, with lower incremental costs in years 4 and 5. The cost contribution of PDC to total costs was low reaching a maximum of $10 \%$ at year 5 .
Fig. 3 Total cost $(€)$ per year for first-line treatment strategies afatinib versus osimertinib. $P D C$ platinum doublet chemotherapy

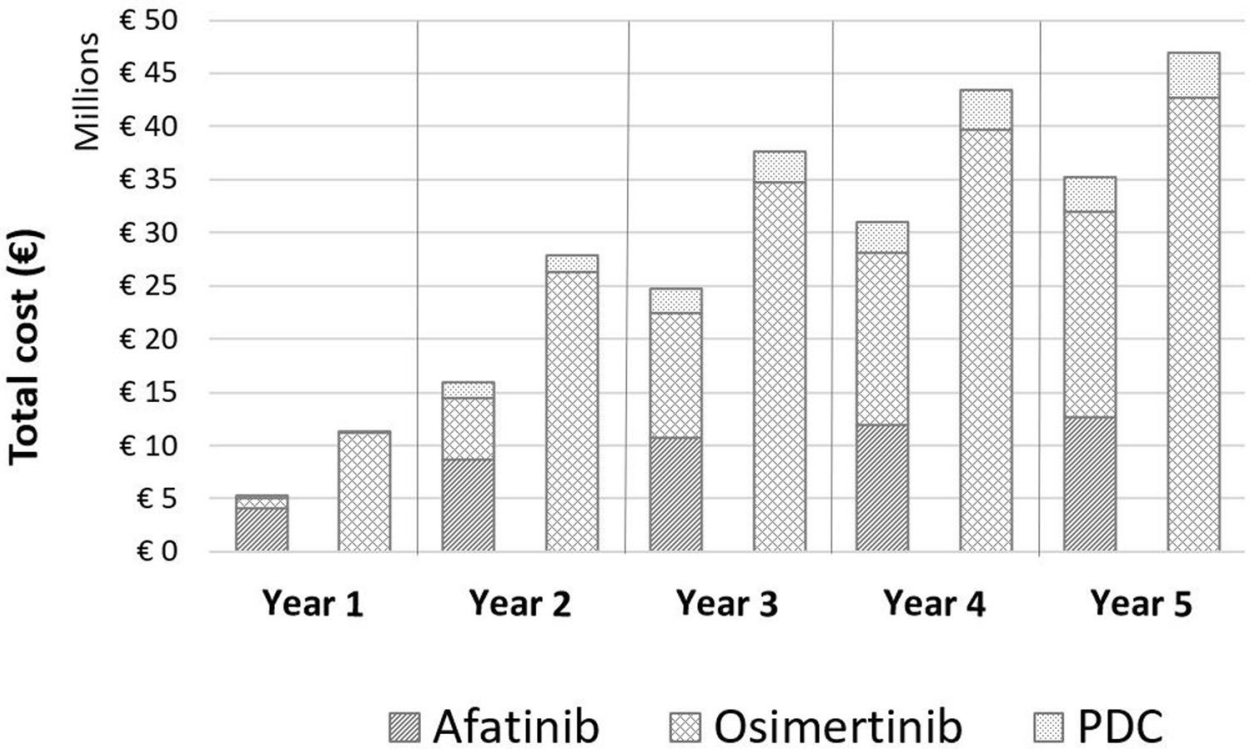


The incremental cost savings were $€ 6.06, € 12.25$ million, $€ 12.26$ million, $€ 9.87$ million and $€ 7.72$ million for years 1, 2, 3, 4 and 5, respectively (see Appendix). Over a 5-year period of time, the incremental budget impact savings of sequential treatment with afatinib versus first-line treatment with osimertinib were estimated to be $€ 48.15$ million.

\section{Scenario analysis}

Results of the two scenario analysis are presented in Table 2. Scenario A, with a log-normal distribution of the survival data showed a similar trend regarding budget impact outcomes to the base-case analysis using the Weibull distribution. The incremental budget impact over a 5-year period yielded cost savings of $€ 54.97$ million when using sequential treatment with afatinib in comparison to first-line osimertinib treatment. The second scenario analysis with $73.1 \%$ proportion of afatinib-treated patients receiving sequential treatment showed incremental budget impact cost savings of $€ 41.34$ million.

\section{Sensitivity analyses}

Figure 4 shows the effect of key parameters on the total annual cost impact over 5 years. The results were most sensitive to the price of osimertinib and afatinib. Results were also sensitive to the percentage of patients with T790M mutation-positive disease receiving subsequent treatment, the percentage of patients testing positive for T790M mutations and the percentage of subsequent treatment after firstline therapy with osimertinib. The costs of adverse events for the different treatments and the cost of T790M mutation testing had only a minor influence on the incremental budget impact.

\section{Discussion}

Over the last decade, various treatment options showed improved PFS data in clinical trials and will support improvement of the burden of disease for lung cancer. The availability of these treatment options has an economic impact, therefore a budget impact analysis was performed to estimate the impact of first-line sequential treatment of afatinib versus the introduction of osimertinib as first-line therapy for NSCLC patients with common EGFR mutations in the Netherlands. The results of this analysis indicated that the incremental budget impact savings of first-line sequential treatment with afatinib in comparison to first-line sequential treatment with osimertinib was $€ 48$ million over a period of 5 years. One-way sensitivity analysis showed the model was robust and the variables tested had no substantial influence on the incremental budget impact. As might be expected, the model was most sensitive to the price of osimertinib and afatinib. It is important to note that although there were differences in the tolerability of these agents, the increased frequency and costs of managing adverse events associated with afatinib were not associated with an increased overall treatment cost compared to osimertinib; afatinib had a lower total average cost per patient. Sensitivity analysis also showed that the costs associated with adverse events had little influence on the incremental budget impact.

The results of the model were also sensitive to the percentage of patients with T790M mutation-positive disease receiving subsequent treatment, the percentage of patients testing positive for $7790 \mathrm{M}$ mutations and the percentage of subsequent treatment after first-line therapy with osimertinib. Indeed, the scenario analysis using a higher percentage for the proportion of patients that continued to receive second-line osimertinib following first-line afatinib resulted in smaller incremental budget impact savings due to a longer mean PFS time on second-line osimertinib and therefore increased drug costs. These results highlight the importance of T790M mutation testing, both for patient outcomes and assessment of treatment costs; further research is necessary to confirm the distribution and outcomes of T790M mutation testing. In addition, testing should already have been performed at the first sign of progression on first-line therapy to prevent deterioration of the performance status related to progressive tumor growth. More data on the subsequent treatment options after first-line osimertinib, as alternatives to PDC, are also needed as there is no evidence to support other treatment approaches at this time.

To our knowledge, this is the first full budget impact analysis of first-line sequential therapy with afatinib in comparison to first-line osimertinib and was based on the best currently available evidence, a validated budget impact model, and included sensitivity and scenario analyses. No prior analysis has compared the budget impact of afatinib to osimertinib in newly diagnosed patients with metastatic NSCLC whose tumors have EGFR deletion 19 or L858R mutations. Recently, the Dutch Healthcare Institute evaluated the budget impact of first-line osimertinib in NSCLC patients with EGFR mutations in the Netherlands over a 3-year horizon showing an estimated incremental budget impact of $€ 18.6$ million to $€ 37.1$ million [31]. This is broadly in agreement with our findings. It should be noted that this analysis considered different treatment paradigms to ours; the first-line osimertinib treatment strategy included thirdline treatment with a PD-(L)1 inhibitor and the comparator arm included treatment with either a first- or second-generation TKI in the first-line setting and included a PD-(L)1 inhibitor third line after PDC or PDC third line after osimertinib second line. Our analysis did not include $\mathrm{PD}(\mathrm{L}) 1$ inhibitors, as this treatment approach is not recommended 
Table 2 Overview of outcomes for base case and scenario analyses of budget impact analysis in sequential firstline strategies afatinib versus osimertinib: number of claims, costs per year and budget impact

\begin{tabular}{|c|c|c|c|c|c|}
\hline & Year 1 & Year 2 & Year 3 & Year 4 & Year 5 \\
\hline \multicolumn{6}{|c|}{ Base case with Weibull distribution for the fitted patient survival data } \\
\hline \multicolumn{6}{|l|}{ Number of claims } \\
\hline \multicolumn{6}{|c|}{ First-line treatment strategy: start with afatinib } \\
\hline Afatinib & 1656 & 3625 & 4491 & 4824 & 4941 \\
\hline Osimertinib & 137 & 882 & 1851 & 2596 & 3030 \\
\hline PDC & 69 & 338 & 576 & 728 & 815 \\
\hline \multicolumn{6}{|c|}{ First-line treatment strategy: start with osimertinib } \\
\hline Osimertinib & 1796 & 4276 & 5495 & 5940 & 6071 \\
\hline PDC & 56 & 400 & 794 & 1,020 & 1,108 \\
\hline \multicolumn{6}{|l|}{ Costs per year $(€)$} \\
\hline \multicolumn{6}{|c|}{ First-line treatment strategy: start with afatinib } \\
\hline Afatinib & $4,149,352$ & $8,954,515$ & $11,066,631$ & $11,880,180$ & $12,165,881$ \\
\hline Osimertinib & 852,936 & $5,471,444$ & $11,470,829$ & $16,078,865$ & $18,767,065$ \\
\hline PDC & 270,545 & $1,289,728$ & $2,183,376$ & $2,750,702$ & $3,064,386$ \\
\hline Total costs & $5,272,832$ & $15,715,687$ & $24,720,828$ & $30,709,746$ & $33,997,331$ \\
\hline \multicolumn{6}{|c|}{ First-line treatment strategy: start with osimertinib } \\
\hline Osimertinib & $11,117,588$ & $26,446,972$ & $33,984,110$ & $36,735,961$ & $37,543,356$ \\
\hline PDC & 217,721 & $1,516,634$ & $2,993,874$ & $3,840,531$ & $4,170,923$ \\
\hline Total costs & $11,335,309$ & $27,963,606$ & $36,977,985$ & $40,576,492$ & $41,714,309$ \\
\hline \multicolumn{6}{|l|}{ Budget impact $(€)$} \\
\hline Afatinib & $4,149,352$ & $8,954,515$ & $11,066,631$ & $11,880,179$ & $12,165,881$ \\
\hline Osimertinib & $-10,262,653$ & $-20,975,528$ & $-22,513,282$ & $-20,657,096$ & $-18,776,291$ \\
\hline PDC & $-52,824$ & $-226,906$ & $-810,507$ & $-1,089,829$ & $-1,106,567$ \\
\hline Total budget impact & $-6,062,477$ & $-12,247,919$ & $-12,257,157$ & $-9,866,746$ & $-7,716,987$ \\
\hline
\end{tabular}

Scenario A: log-normal distribution for the fitted patient survival data Number of claims

First-line treatment strategy: start with afatinib

$\begin{array}{lcclll}\text { Afatinib } & 1652 & 3498 & 4369 & 4839 & 5120 \\ \text { Osimertinib } & 140 & 944 & 1886 & 2617 & 3134 \\ \text { PDC } & 70 & 367 & 612 & 766 & 862 \\ \text { First-line treatment strategy: start } & \text { with osimertinib } & & & \\ \text { Osimertinib } & 1802 & 4250 & 5622 & 6413 & 6899 \\ \text { PDC } & 54 & 410 & 774 & 997 & 1125\end{array}$

Costs per year $(€)$

First-line treatment strategy: start with afatinib

\begin{tabular}{|c|c|c|c|c|c|}
\hline Afatinib & $4,139,458$ & $8,644,910$ & $10,770,102$ & $11,917,980$ & $12,602,145$ \\
\hline Osimertinib & 876,051 & $5,857,182$ & $11,687,707$ & $16,211,293$ & $19,405,814$ \\
\hline PDC & 276,751 & $1,395,414$ & $2,314,444$ & $2,888,088$ & $3,233,655$ \\
\hline Total costs & $5,292,261$ & $15,897,506$ & $24,722,253$ & $31,017,361$ & $35,241,615$ \\
\hline \multicolumn{6}{|c|}{ First-line treatment strategy: start with osimertinib } \\
\hline Osimertinib & $11,160,108$ & $26,291,129$ & $34,767,384$ & $39,659,294$ & $42,662,810$ \\
\hline PDC & 208,204 & $1,553,835$ & $2,915,326$ & $3,746,964$ & $4,221,615$ \\
\hline Total costs & $11,368,311$ & $27,844,964$ & $37,682,710$ & $43,406,257$ & $46,884,425$ \\
\hline \multicolumn{6}{|l|}{ Budget impact (€) } \\
\hline Afatinib & $4,139,458$ & $8,644,910$ & $10,770,102$ & $11,917,980$ & $12,602,145$ \\
\hline Osimertinib & $-10,284,056$ & $-20,433,947$ & $-23,079,677$ & $-23,448,011$ & $-23,256,996$ \\
\hline PDC & 68,548 & $-158,421$ & $-600,881$ & $-858,876$ & $-987,960$ \\
\hline Total budget impact & $-607,651$ & $-11,947,458$ & $-12,910,457$ & $-12,388,897$ & $-11,642,810$ \\
\hline
\end{tabular}


Table 2 (continued)

\begin{tabular}{|c|c|c|c|c|c|}
\hline & Year 1 & Year 2 & Year 3 & Year 4 & Year 5 \\
\hline \multicolumn{6}{|c|}{ Scenario B: alternative proportion of afatinib treated patients receiving sequential treatment $(\mathbf{7 3 . 1 \% )}$} \\
\hline \multicolumn{6}{|l|}{ Number of claims } \\
\hline \multicolumn{6}{|c|}{ First-line treatment strategy: start with afatinib } \\
\hline Afatinib & 1656 & 3625 & 4491 & 4824 & 4941 \\
\hline Osimertinib & 159 & 1023 & 2148 & 3012 & 3516 \\
\hline PDC & 51 & 258 & 464 & 615 & 711 \\
\hline \multicolumn{6}{|c|}{ First-line treatment strategy: start with osimertinib } \\
\hline Osimertinib & 1796 & 4276 & 5495 & 5940 & 6071 \\
\hline PDC & 56 & 400 & 794 & 1020 & 1108 \\
\hline \multicolumn{6}{|l|}{ Costs per year $(€)$} \\
\hline \multicolumn{6}{|c|}{ First-line treatment strategy: start with afatinib } \\
\hline Afatinib & $4,149,352$ & $8,954,515$ & $11,066,631$ & $11,880,180$ & $12,165,881$ \\
\hline Osimertinib & 989,676 & $6,348,612$ & $13,309,803$ & $18,656,587$ & $21,775,753$ \\
\hline PDC & 200,535 & 984,161 & $1,758,926$ & $2,321,211$ & $2,662,846$ \\
\hline Total costs & $5,339,563$ & $16,287,288$ & $26,135,360$ & $32,857,978$ & $36,604,479$ \\
\hline \multicolumn{6}{|c|}{ First-line treatment strategy: start with osimertinib } \\
\hline Osimertinib & $11,117,588$ & $26,446,972$ & $33,984,110$ & $36,735,961$ & $37,543,356$ \\
\hline PDC & 217,721 & $1,516,634$ & $1,993,874$ & $3,840,531$ & $4,170,953$ \\
\hline Total costs & $11,335,309$ & $27,963,606$ & $36,977,985$ & $40,576,492$ & $41,714,309$ \\
\hline \multicolumn{6}{|l|}{ Budget impact $(€)$} \\
\hline Afatinib & $4,149,352$ & $8,954,515$ & $11,066,631$ & $11,880,179$ & $12,165,881$ \\
\hline Osimertinib & $-10,127,912$ & $-20,098,360$ & $-20,674,307$ & $-18,079,373$ & $-15,767,603$ \\
\hline PDC & $-17,186$ & $-532,473$ & $-1,234,948$ & $-1,519,320$ & $-1,508,107$ \\
\hline $\begin{array}{l}\text { Total incremental budget } \\
\text { impact }\end{array}$ & $-5,995,746$ & $-11,676,318$ & $-10,842,624$ & $-7,718,514$ & $-5,109,829$ \\
\hline
\end{tabular}

$P D C$ platinum doublet chemotherapy

by ESMO or Dutch guidelines for patients with EGFR-activating mutations [2, 32].

Further cost-effectiveness analysis is necessary to confirm our cost and quality-of-life assessment comparison between first-line sequential treatment with afatinib versus first-line treatment with osimertinib. The cost-effectiveness of osimertinib has previously been evaluated in Canada [33], the United States and Brazil [34]. In the analysis conducted in Canada, first-line osimertinib therapy was compared to EGFR TKIs gefitinib and afatinib and was not considered as a cost-effective treatment option at a willingness-to-pay threshold of $\$ 100,000$ per QALY [33]. Similar results were reported in the United States and Brazil using World Health Organization cost-effectiveness threshold criteria; at current costs osimertinib was unlikely to be cost-effective in either the United States or Brazil [34]. The clinical benefit of firstline osimertinib over alternatives will likely carry the greatest weight in funding decisions [33].

There are several limitations that should be considered. As no data were available on the efficacy and median PFS of PDC as third-line treatment, data from the second-line setting of the IMPRESS trial were used which likely overestimated the treatment benefit in this treatment arm and also overestimated the additional costs coming from this treatment benefit. To avoid an over- or underestimation on the quality of life of treatments resulting from the exclusion of adverse events for which disutility data were not available, assumptions were made for dyspnea, leukopenia, paronychia and stomatitis. Further research regarding disutilities of adverse events is needed to support these assumptions. The intention of our model was to reconstruct the patients' time on treatment per first-, second-, and third-line of treatment based on the clinical trials for afatinib, osimertinib and PDC, respectively. As no direct head-to-head studies have compared afatinib versus osimertinib, this model used a mixed treatment model approach. One of the largest differences between patient populations was the presence of the T790-positive mutation in all included EGFR-positive NSCLC patients in the AURA3 trial. The inclusion of these data had to be taken into account in our survival model to be able to reconstruct the effect of second-line treatment with osimertinib. The reason was that in the AURA3 trial the efficacy of second-line osimertinib was evaluated for this specific indication.

Even though the included RCTs were overall well balanced, we are aware of potential selection bias due to 


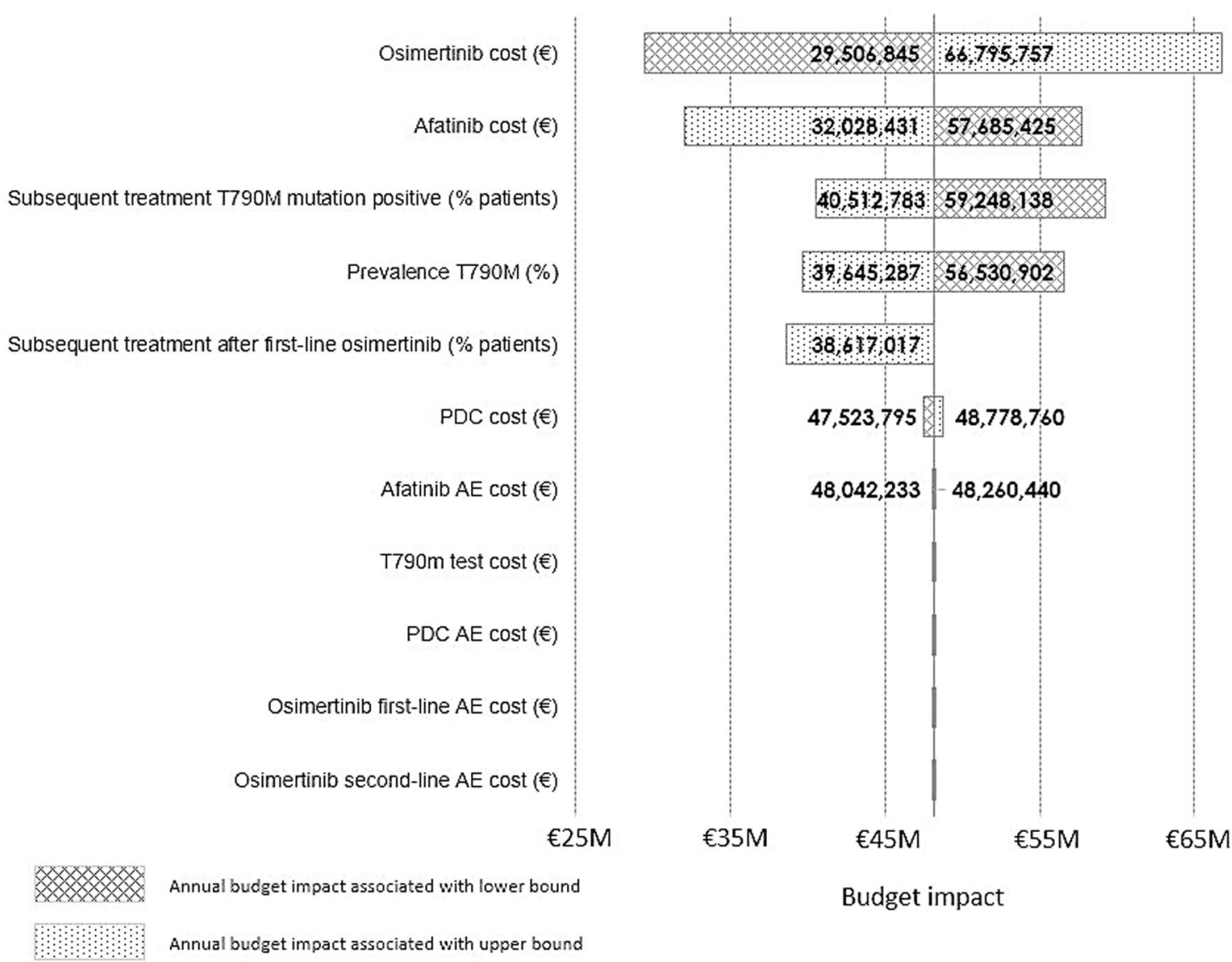

Fig. 4 One-way sensitivity analysis: impact of changes of input parameters on the change in total annual budget in year $5 . A E$ adverse event, $P D C$ platinum doublet chemotherapy

differences in patient populations such as age, race, smoking history, EGFR mutations status and presence of T790M positive mutation, resulting in potential over- or underestimation for both treatment arms.

The model did not include any other second- or thirdline treatment options than PDC therapy or second-line osimertinib; it should be recognized that the inclusion of other emerging therapies would alter budget impact and total costs of both treatment strategies. Further research is needed with a head-to-head comparison of osimertinib versus afatinib and other second-generation TKIs in a clinical trial or realworld data setting to support the outcomes of this budget impact model. Finally, compliance was assumed to be $100 \%$ for all treatments which may not reflect real-world practice. This may overestimate treatment costs and would also affect the clinical benefits of treatment.

In conclusion, the use of afatinib as a first-line treatment approach would have a substantial lower financial impact on the healthcare budget in the Netherlands and would lead to a higher incremental time on treatment of 4.5 months and an incremental QALM of 2.8 compared to a sequential treatment approach starting with osimertinib. The total budget impact over 5 years was $€ 110.4$ million for the sequence starting with afatinib versus $€ 158.6$ million for the sequence starting with osimertinib, leading to a total incremental cost saving of $€ 48.15$ million. Together with clinical data, medicine prices and market access agreements will support governments and healthcare payers to decide how to manage the worldwide leading cause of cancer mortality.

Acknowledgements The authors thank Sue Patel, whose work was funded by Boehringer Ingelheim Inc. for medical writing assistance in the preparation of this manuscript.

Author contributions LW, JN, CS, MP contributed to the concept and design and to the acquisition and statistical analysis of the data. All authors contributed to the analysis and interpretation of data and to the drafting and revision of the manuscript.

Funding This study was funded by Boehringer Ingelheim GmbH, Alkmaar, The Netherlands.

Data availability The data used in this research are not confidential since they were obtained from publicly available sources. 


\section{Compliance with ethical standards}

Conflict of interest MP received grants and honoraria from various pharmaceutical companies, including other companies interested in the subject of this paper. LW is employee of Asc Academics Inc. IG and $\mathrm{JN}$ are employees of Boehringer Ingelheim. CS is a former employee of Boehringer Ingelheim and owns Outcomes Analytica AS.

Research involving human participants and/or animals For this study all used research data were obtained from publicly available sources, no new studies with human participants and/or animals were performed.

Informed consent Since all research data were obtained from publicly available sources and no new studies involving human participants were performed, there was no need for consent for publication.

Open Access This article is licensed under a Creative Commons Attribution 4.0 International License, which permits use, sharing, adaptation, distribution and reproduction in any medium or format, as long as you give appropriate credit to the original author(s) and the source, provide a link to the Creative Commons licence, and indicate if changes were made. The images or other third party material in this article are included in the article's Creative Commons licence, unless indicated otherwise in a credit line to the material. If material is not included in the article's Creative Commons licence and your intended use is not permitted by statutory regulation or exceeds the permitted use, you will need to obtain permission directly from the copyright holder. To view a copy of this licence, visit http://creativecommons.org/licenses/by/4.0/.

\section{References}

1. Bray, F., Ferlay, J., Soerjomataram, I., Siegel, R.L., Torre, L.A., Jemal, A.: Global cancer statistics 2018: GLOBOCAN estimates of incidence and mortality worldwide for 36 cancers in 185 countries. CA Cancer J. Clin. 68, 394-424 (2018)

2. Planchard, D., Popat, S., Kerr, K., Novello, E., Smit, F., FaivreFinn, C., et al.: Metastatic non-small cell lung cancer: ESMO Clinical Practice Guidelines for diagnosis, treatment and followup. Ann. Oncol. 29, iv192-iv237 (2018)

3. Maemondo, M., Inoue, A., Kobayashi, K., Sugawara, S., Oizumi, S., Isobe, H., et al.: Gefitinib or chemotherapy for non-small-cell lung cancer with mutated EGFR. N. Engl. J. Med. 362, 2380$2388(2010)$

4. Mitsudomi, T., Morita, S., Yatabe, Y., Negoro, S., Okamoto, I., Tsurutani, J., et al.: Gefitinib versus cisplatin plus docetaxel in patients with non-small-cell lung cancer harbouring mutations of the epidermal growth factor receptor (WJTOG3405): an open label, randomised phase 3 trial. Lancet Oncol. 11, 121-128 (2010)

5. Rosell, R., Carcereny, E., Gervais, R., Vergnenegre, A., Massuti, B., Felip, E., et al.: Erlotinib versus standard chemotherapy as first-line treatment for European patients with advanced EGFR mutation-positive non-small-cell lung cancer (EURTAC): a multicentre, open-label, randomised phase 3 trial. Lancet Oncol. 13, 239-246 (2012)

6. Han, J.Y., Park, K., Kim, S.W., Lee, D.H., Kim, H.Y., Kim, H.T., et al.: First-SIGNAL: first-line single-agent IRESSA versus gemcitabine and cisplatin trial in never-smokers with adenocarcinoma of the lung. J. Clin. Oncol. 30, 1122-1128 (2012)

7. Inoue, A., Kobayashi, K., Maemondo, M., Sugawara, S., Oizumi, S., Isobe, H., et al.: Updated overall survival results from a randomized phase III trial comparing gefitinib with carboplatinpaclitaxel for chemo-naïve non-small cell lung cancer with sensitive EGFR gene mutations (NEJ002). Ann. Oncol. 24, 54-59 (2013)

8. Sequist, L.V., Yang, J.C., Yamamoto, N., O’Byrne, K., Hirsch, V., Mok, T., et al.: Phase III study of afatinib or cisplatin plus pemetrexed in patients with metastatic lung adenocarcinoma with EGFR mutations. J. Clin. Oncol. 31, 3327-3334 (2013)

9. Wu, Y.L., Zhou, C., Hu, C.P., Feng, J., Lu, S., Huang, Y., et al.: Afatinib versus cisplatin plus gemcitabine for first-line treatment of Asian patients with advanced non-small-cell lung cancer harbouring EGFR mutations (LUX-Lung 6): an open-label, randomised phase 3 trial. Lancet Oncol. 15, 213-222 (2014)

10. Park, K., Tan, E.H., O’Byrne, K., Zahng, L., Boyer, M., Mok, T., et al.: Afatinib versus gefitinib as first-line treatment of patients with EGFR mutation-positive non-small-cell lung cancer (LUXLung 7): a phase 2B, open-label, randomised controlled trial. Lancet Oncol. 17, 577-589 (2016)

11. Wu, Y.L., Cheng, Y., Zhou, X., Lee, K.H., Nakagawa, K., Niho, S., et al.: Dacomitinib versus gefitinib as first-line treatment for patients with EGFR-mutation-positive non-small-cell lung cancer (ARCHER 1050): a randomised, open-label, phase 3 trial. Lancet Oncol. 18, 1454-1466 (2017)

12. Soria, J.C., Ohe, Y., Vansteenkiste, J., Reungwetwattana, T., Chewaskulyong, B., Hyeong Lee, K., Dechaphunkul, A., et al.: Osimertinib in untreated EGFR-mutated advanced non-small-cell lung cancer. N. Engl. J. Med. 378, 113-125 (2018)

13. Mok, T.S., Wu, Y.L., Ahn, M.J., Garassino, M.C., Kim, H.R., Ramalingam, S.S., et al.: Osimertinib or platinum-pemetrexed in EGFR T790M-positive lung cancer. N. Engl. J. Med. 376, 629640 (2017)

14. Ramalingam, S.S., Yang, J.C., Lee, C.K., Kurata, T., Kim, D.W., John, T., et al.: Osimertinib as first-line treatment of EGFR mutation-positive advanced non-small-cell lung cancer. J. Clin. Oncol. 36, 841-849 (2018)

15. Girard, N.: Optimizing outcomes in EGFR mutation-positive NSCLC: which tyrosine kinase inhibitor and when? Future Oncol. 14, 1117-1132 (2018)

16. Hirsh, V.: Turning EGFR mutation-positive non-small-cell lung cancer into a chronic disease: optimal sequential therapy with EGFR tyrosine kinase inhibitors. Ther. Adv. Med. Oncol. 10, 1758834017753338 (2018)

17. Sequist, L., Wu, Y.-L., Schuler, M., Kato, T., Yang, J.C., Tanaka, H., et al.: Subsequent therapies post-afatinib among patients (pts) with EGFR mutation-positive (EGFRm+) NSCLC in LUX-Lung (LL) 3, 6 and 7. Ann. Oncol. 28 (2017)

18. Hochmair, M.J., Morabito, A., Hao, D., Yang, C.T., Soo, R.A., Yang, J.C., et al.: Sequential treatment with afatinib and osimertinib in patients with EGFR mutation-positive non-small-cell lung cancer: an observational study. Future Oncol. 14, 2861-2874 (2018)

19. Volksgezondheidenzorg.info: Contribution of lung cancer to disease burden, government website (2019). https://www.volksgezon dheidenzorg.info/onderwerp/longkanker/cijfers-context/ziektelast \#node-bijdrage-van-longkanker-aan-ziektelast. Accessed 2 Oct 2018

20. Integrated Cancer center the Netherlands (IKNL), 2017, Dutch Cancer registration, Available: https://www.cijfersoverkanker.nl/ selecties/Dataset_1/img5af2af2a2b91e, October 2,2018.

21. Sullivan, S.D., Mauskopf, J.A., Augustovski, F., Jaime Caro, J., Lee, K.M., Minchin, M., et al.: Budget impact analysis-principles of good practice: report of the ISPOR 2012 Budget Impact Analysis Good Practice II Task Force. Value Health. 17, 5-14 (2014) 
22. Soria, J.C., Wu, Y.L., Nakagawa, K., Kim, S.W., Yang, J.J., Ahn, M.J., et al.: Gefitinib plus chemotherapy versus placebo plus chemotherapy in EGFR-mutation-positive non-small-cell lung cancer after progression on first-line gefitinib (IMPRESS): a phase 3 randomised trial. Lancet Oncol. 16, 990-998 (2015)

23. Guyot, P., Ades, A., Ouwens, M.J., Welton, N.J.: Enhanced secondary analysis of survival data: reconstructing the data from published Kaplan-Meier survival curves. BMC Med. Res. Methodol. 12, 9 (2012)

24. Hochmair, M.J., Buder, A., Schwab, S., Burghuber, O.C., Prosch, H., Hilbe, W., et al.: Liquid-biopsy-based identification of EGFR T790M mutation-mediated resistance to afatinib Treatment in patients with advanced EGFR mutation-positive NSCLC, and subsequent response to osimertinib. Target Oncol. 14, 75-83 (2019)

25. Yu, H.A., Arcila, M.E., Rekhtman, N., Sima, C.S., Zakowsli, M.F., Pao, W., et al.: Analysis of tumor specimens at the time of acquired resistance to EGFR-TKI therapy in 155 patients with EGFR-mutant lung cancers. Clin. Cancer Res. 19, 2240-2247 (2013)

26. Yang, J.C., Wu, Y.L., Schuler, M., Sebastian, M., Popat, S., Yamamoto, N., et al.: Afatinib versus cisplatin-based chemotherapy for EGFR mutation-positive lung adenocarcinoma (LUX-Lung 3 and LUX-Lung 6): analysis of overall survival data from two randomised, phase 3 trials. Lancet Oncol. 16, 141-151 (2015)

27. Nafees, B., Stafford, M., Gavriel, S., Bhalla, S., Watkins, J.: Health state utilities for non small cell lung cancer. Health Qual. Life Outcomes. 6, 84 (2008)

28. Chouaid, C., Agulnik, J., Goker, E., Herder, G.J., Lester, J.F., Vansteenkiste, J., et al.: Health-related quality of life and utility in patients with advanced non-small-cell lung cancer: a prospective cross-sectional patient survey in a real-world setting. J. Thorac. Oncol. 8, 997-1003 (2013)

29. Kohsaka, S., Petronczki, M., Solca, F., Maemondo, M.: Tumor clonality and resistance mechanisms in EGFR mutation-positive non-small-cell lung cancer: implications for therapeutic sequencing. Future Oncol. 15, 637-652 (2018)

30. Claxton, K.: The irrelevance of inference: a decision-making approach to the stochastic evaluation of health care technologies. J. Health Econ. 18(3), 341-364 (1999)

31. Wijma, S.: Healthcare Insitute the Netherlands; Package advice osimertinib (Tagrisso) for first line treatment of patients with advanced or metastatic non-small-cell lung cancer (NSCLC) with activating EGFR-mutations, Dutch Osimertinib dossier for Ministry of Health, Ref.nr: 2018051432 (2018)
32. Guideline non-small-cell lung cancer version, National workstream lung tumours (2015). https://www.oncoline.nl/niet-klein cellig-longcarcinoom. Accssed 15 Jan 2019

33. Ezeife, D.A., Kirk, V., Chew, D.S., Nixon, N.A., Lee, R., Le, L.W., et al.: Economic analysis of osimertinib in previously untreated EGFR-mutant advanced non-small cell lung cancer in Canada. Lung Cancer. 125, 1-7 (2018)

34. Aguiar Jr., P.N., Haaland, B., Park, W., San Tan, P., Del Giglio, A., de Lima, G., Jr., : Cost-effectiveness of osimertinib in the first-line treatment of patients with EGFR-mutated advanced non-small cell lung cancer. JAMA Oncol. 4, 1080-1084 (2018)

35. Healthcare Institute the Netherlands: The price for a 30-day prescription of afatinib (2018). https://www.medicijnkosten.nl/datab ank?zoekterm $=$ afatinib\&toedieningsvorm $=$ TABLETTEN $\% 20$ EN\%20CAPSULES\&ddd_per_artikel=0\&hoeveelheid=30,00. Accessed 11 Dec 2018

36. G-Standard: Dutch treatment database (Z-index), October 2018 (2018)

37. National Institute for Health and Care Excellence: Plasma EGFR mutation tests for adults with locally advanced or metastatic nonsmall-cell lung cancer (MIB137) (2018). https://www.niceorguk/ advice/mib137

38. Uyl-de Groot, C.A.M.J., Zaim, R.: Nivolumab (Opdivo) bij Gevorderd Plaveiselcelcarcinoom van de Long. Instituut Beleid \& Management Gezondheidszorg (2015)

39. Wehler, E., Zhao, Z., Pinar Bilir, S., Munakata, J., Barber, B.: Economic burden of toxicities associated with treating metastatic melanoma in eight countries. Eur. J. Health Econ. 18, 49-58 (2017)

40. Mickisch, G., Gore, M., Escudier, B., Procopio, G., Walzer, S., Nuiten, M.: Costs of managing adverse events in the treatment of first-line metastatic renal cell carcinoma: bevacizumab in combination with interferon-alpha2a compared with sunitinib. Br. J. Cancer. 102, 80-86 (2010)

41. Hakkaart-van Roijen, L., Van der Linden, N., Bouwmans, C., Kanters, T., Swan, T.S.: Cost manual, Methodology for cost research and reference pricing for economic evaluations in healthcare. Paragraph 3.1.2.8; Outpatient setting (2015)

Publisher's Note Springer Nature remains neutral with regard to jurisdictional claims in published maps and institutional affiliations. 